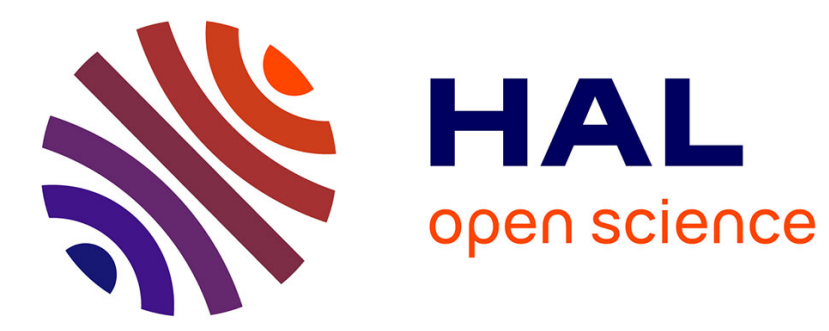

\title{
Les avatars du mot diaspora: du lien créé par le malheur commun à une catégorie de l'action publique
}

Jacques Barou

\section{To cite this version:}

Jacques Barou. Les avatars du mot diaspora: du lien créé par le malheur commun à une catégorie de l'action publique. Sens-Dessous, 2018. halshs-01761222

\section{HAL Id: halshs-01761222 \\ https://shs.hal.science/halshs-01761222}

Submitted on 8 Apr 2018

HAL is a multi-disciplinary open access archive for the deposit and dissemination of scientific research documents, whether they are published or not. The documents may come from teaching and research institutions in France or abroad, or from public or private research centers.
L'archive ouverte pluridisciplinaire HAL, est destinée au dépôt et à la diffusion de documents scientifiques de niveau recherche, publiés ou non, émanant des établissements d'enseignement et de recherche français ou étrangers, des laboratoires publics ou privés. 


\section{Les avatars du mot diaspora : du lien créé par le malheur commun à une catégorie de l'action publique}

Le terme diaspora est d'un usage de plus en plus répandu pour désigner des populations immigrées ou issues d'immigrations plus ou moins anciennes. Cet usage «commun » d'un concept forgé par plusieurs décennies de recherche académique dans le champ des sciences humaines est révélateur des évolutions du monde contemporain. Initialement, on distinguait d'une part, des diasporas «classiques » inspirées du modèle de la diaspora juive, définies autour de l'idée d'un peuple dispersé dont la conscience unitaire se serait maintenue par de delà les effets dévastateurs de la séparation grâce au lien, imaginaire ou réel, entretenu avec le territoire d'origine et d'autre part les diaspora «hybrides » construites autour de références multiples et d'un principe de mobilité qui implique un changement permanent dans la construction des identités. Selon le sociologue britannique Paul Gilroy, ce second modèle correspondrait à l'identité diasporique des populations noires des Amériques ${ }^{1}$. Dans le premier cas, il s'agirait de diasporas centrées, constituées autour d'une racine unique. Dans le second cas, il s'agirait de diasporas où, selon les termes de Richard Marienstras, «le centre est partout et la circonférence nulle part $»^{2}$. Plutôt que de se référer à une racine unique, elles se construiraient sur le modèle du rhizome, privilégiant les réseaux des liens et des échanges entre plusieurs localisations.

Cette seconde acceptation correspond davantage au sens premier du terme diaspora. Etymologiquement, le mot renvoie à un processus qui relève de la botanique. Il s'agit de la dispersion des spores, des graines de diverses plantes. La traduction française la plus fidèle à cette étymologie serait le terme : ensemencement. Une plante dont les semences ont été prélevées en un lieu précis est semée dans divers lieux qui offrent des conditions différentes à sa croissance et aboutissent à la production de variétés locales différentes les unes des autres mais conservant toutes les principales caractéristiques de la plante originelle.

\section{Le rôle du centre et de la tragédie}

Pris dans ce sens le terme diaspora est porteur d'une vision relativement optimiste de l'avenir. Le peuple chassé de son pays par diverses causes : violence, pauvreté, calamités naturelles a toujours la possibilité de revivre ailleurs en se modifiant certes mais en conservant l'essence de son identité. Mais il lui faut cultiver le lien avec la souche commune pour garder conscience de ce qui peut unir les fractions dispersées qui peuvent toujours se fondre dans le nouveau paysage. C'est là un point commun entre les diasporas classiques et les diasporas hybrides. Si la diaspora juive a été le modèle du premier type, c'est avant tout parce que la religion, institution par excellence permettant le maintien du lien, a pu rappeler sans cesse l'origine commune des communautés dispersées. Elle l'a fait à travers l'entretien du souvenir du malheur en toutes circonstances, comme dans le cas de la coutume qui veut que les jeunes mariés brisent le verre dans lequel ils viennent de boire pour se remémorer, en ce moment de bonheur, les destructions tragiques des deux temples de Jérusalem. Elle l'a fait aussi à travers la sacralisation de l'espérance du retour faisant de celle-ci une véritable obligation pour les

\footnotetext{
${ }_{1}^{1}$ P. Gilroy, L’atlantique noir, modernité et double consciences, éditions Amsterdam, 2010, 333p.

${ }^{2}$ R.Marienstrass, Pour la Diaspora, Le débat, n¹44, avril-mai 2007
} 
fidèles comme l'exprime le psaume 137 récité dans plusieurs circonstances et qui renvoie à l'exil subi à Babylone après la destruction du premier temple :

«Si je t'oublie Jérusalem, que ma main droite se dessèche,

Que ma langue s'attache à mon palais, si je ne me souviens de toi, si je ne fais de Jérusalem le principal sujet de ma joie ! ${ }^{3}$

On est ici dans l'archétype de la diaspora centrée. Jérusalem, ville martyr est le point vers lequel tous les Juifs dispersés ne doivent jamais cesser de porter leur regard et de diriger leurs espérances.

Il n'y a pas d'exemple aussi fort de diaspora tournant autour d'un centre aussi valorisé parmi les populations qui ont du se disperser à la suite d'une tragédie. Le messianisme qui est au cœur du judaïsme et les persécutions récurrentes subies par les diverses diasporas juives pendant plusieurs siècles ne sont bien sûr pas étrangers à cette référence à un centre paré d'autant de sacralité.

Dans d'autres diasporas, s'il y a bien un désir de retour qui sert à renforcer la part d'identité commune aux populations dispersées, il n'est jamais aussi fortement relié à un centre aux contours aussi précis. La diaspora grecque a commencé dès l'antiquité par l'établissement de colonies de peuplement en divers points de la méditerranée fondées par différentes cités, telle Phocée, elle-même colonie athénienne d'Asie Mineure qui fonda Massalia l'actuelle Marseille ainsi que plusieurs villes sur les côtes de la méditerranée occidentale. Avec l'empire byzantin, elle prend une dimension encore plus large. Le terme d'omogénia littéralement « de même race » utilisé en grec pour désigner cette diaspora souligne le lien qui doit persister. La diaspora qui se fait alors dans un processus de conquête et de colonisation plus ou moins pacifique est plutôt l'expression d'un essaimage lié à la puissance maritime et politique de la Grèce continentale et son extension dans l'ouest de l'Asie mineure.

A partir du 29 mai 1453, date de la prise de Constantinople par les Turcs et la fin de l'empire byzantin, le mot change de contenu. Il renvoie à l'exil des vaincus, un exil qui s'étale sur une longue période avec en particulier l'échange de populations organisé par le traité de Lausanne de 1924 qui voit 1,3 million de Grecs de Turquie quitter un pays où ils vivaient depuis toujours pour s'installer dans une terre ancestrale dont beaucoup ne parlaient pas la langue. Cet échange résultait de l'issue de la guerre engagée en 1919 par la Grèce pour tenter de reconquérir ses anciennes possessions byzantines au nom de la «mégali idea » la grande idée de réunion des Grecs autochtones et des Grecs allochtones qui se trouvaient dans divers pays des Balkans, dans les villes de l'ouest de la Turquie et en Anatolie. Cette grande idée de constituer un état nation fusionnant le pays d'origine et la diaspora des pays voisins a été une constante de la politique étrangère grecque depuis l'indépendance en 1830 jusqu'à la dictature des colonels dans les années 1970. Elle n'est pas seulement une forme de nationalisme extensif comme pouvaient l'être le pangermanisme et le panslavisme au XIXe et au XXe siècle. Elle s'appuie d'abord sur l'entretien d'une nostalgie de la période byzantine dans

\footnotetext{
${ }^{3}$ La Bible de Jérusalem, Livre des psaumes, p 1026, Le Cerf, Paris.
} 
laquelle Constantinople joue un rôle un peu comparable à celui de Jérusalem pour les Juifs mais non pas en tant que pôle unique de la civilisation grecque mais en tant que phare de l'Hellénisme, cette expansion civilisatrice de la Grèce en méditerranée comme le dit explicitement le premier ministre Ioannis Kolettis en 1844: "Il y a deux grands centres de l'Hellénisme: Athènes est la capitale du royaume. Constantinople est la grande capitale, la Ville, l'espoir de tous les Grecs ». En fait le but est moins un retour à Constantinople que la reconstitution de la «Grande Grèce » de l'antiquité. Il s'agit de transformer une diaspora dominée dans les pays voisins en une diaspora réunifiée reprenant flatteusement son rôle civilisateur comme le prônaient certains théoriciens de la mégali idea. Le premier ministre Kolletis déclarait aussi en 1844 : "Ayant l'Orient à sa droite et l'occident à sa gauche, la Grèce est prédestinée par sa renaissance à éclairer l'Orient comme elle le fut par son essor à éclairer l'occident. ». La défaite militaire de la Grèce en 1923 devant les troupes turques sonna le glas de cette idée, peu réaliste, d'unir politiquement et territorialement le pays d'origine et une diaspora installée depuis des siècles dans des pays où elle se serait sans doute fondue complètement si elle n'avait pas subi différentes discriminations et persécutions de la part des majorités. La mégali idea disparaitra des références politiques après la crise de Chypre en 1974, ultime tentative d'unir la Grèce continentale avec une population insulaire d'origine grecque.

D'autres diasporas comme celle des Arméniens ou des Palestiniens ont connu un devenir semblable. Elles continuent d'exister indépendamment de l'existence ou de l'absence d'un Etat de référence.

Culture du souvenir, sacralisation des lieux habités dans le passé, attention portée aux souffrances de certaines communautés de la diaspora, voire désir de revanche sont les principales composantes du lien qui unit les diaspora classiques qui jusqu'à l'époque contemporaine rapproche intellectuellement et affectivement les populations dispersées à la suite de diverses tragédies.

\section{Multiplicités des références}

Bien que la tragédie, en l'occurrence la traite esclavagiste, soit aussi à l'origine des diasporas africaines constituées dans l'espace américain, elle joue de manière générale un rôle moindre dans l'entretien du lien transnational. Avec ses quarante-six états nations et ses milliers de groupes ethniques dispersés à travers différents pays, l'Afrique apparaît comme peu propice à inspirer à ceux qui la quittent, un sentiment d'unité et un intérêt soutenu envers la terre ancestrale. On observe pourtant un usage récurrent de la notion de diaspora africaine ou, plus encore, de diaspora noire dans le langage courant et particulièrement dans le langage militant. Les références à la diaspora noire comme une réalité présentant une certaine unité se retrouvent essentiellement dans les discours produits par une élite intellectuelle et politique dont rien ne permet d'affirmer qu'elle est représentative d'un sentiment majoritaire dans l'opinion publique. C'est ce qu'écrit Christine Chivallon à propos des thèses de Paul Gilroy sur la «Black Atlantic » ${ }^{4}$, cette diaspora afro-américaine qui correspondrait à un modèle

\footnotetext{
${ }^{4}$ Paul Gilroy, The Black Atlantic. Modernity and Double Consciousness. London,Verso, 1993, 261 p.
} 
unique, celui de l'hybridité : "on peut se demander si le choix de Gilroy consistant à privilégier le discours de quelques figures de la sphère politique et littéraire, était le bon. Que dire d'une sphère plus " ordinaire », celle où se construit le " culturel » au quotidien? N'estce pas là que se définissent les termes d'une appartenance sociale dont le discours politique et littéraire n'est qu'une des modalités de mise en représentation? »5

Cette auteure, spécialiste de la Caraïbe, pense que le modèle hybride n'est que l'un des trois modèles théoriques permettant de rendre compte du contenu de la diaspora noire dans les Amériques. Les diasporas hybrides se définissent non pas par la continuité et le mythe d'un retour vers un centre mais au contraire par la diversité, le métissage, la dissémination. Elles correspondent à un processus de «créolisation », c'est-à-dire d'invention d'une culture nouvelle faite d'échanges et de métissages. Si le lien avec l'Afrique est présent, il n'est pas exclusif d'autres liens en particulier avec le monde du proche environnement qui est à dominante européenne mais peut aussi comporter des éléments amérindiens et asiatiques.

Il y a aussi des diasporas noires qui s'aliènent totalement et subissent un processus de déculturation sans pouvoir parfois acquérir une autre culture. Elles se marginalisent et ne se définissent plus par une culture, que celle-ci soit homogène ou faite d'emprunts multiples mais par une position d'exclus. Elles n'existent plus en tant que groupe culturel mais en tant que milieu social déclassé, milieu sans mémoire n'entretenant aucun lien diasporique avec une origine réelle ou fantasmée. Bien qu'issues d'un exil tragique, ces populations ne peuvent plus être qualifiées de diaspora dans la mesure où elles n'ont conscience d'aucun lien avec un territoire d'origine ni avec des communautés de même provenance.

Il y a enfin un modèle de diaspora noire qui évoque les diasporas classiques avec une volonté de créer une continuité avec l'Afrique. Comme il n'y a pas de point précis à désigner sur le continent, il s'agit souvent d'une Afrique mythifiée, parée de tous les attraits et de toutes les richesses et qui n'aurait été affaiblie que par la rapacité du monde «blanc ». L'insistance sur la maltraitance subie par le passé dans le cadre de la traite atlantique et dans l'économie de plantation encourage parfois à un nationalisme qui peut prendre un contenu raciste. Cela peut correspondre aux dérives de certains groupes radicaux en particulier aux Etats-Unis.

Toutefois, le lien avec le pays d'origine, et même avec un centre relativement précis peut se construire en dehors de toute idéologie politique. Il se manifeste en particulier au Brésil ou à Cuba à travers les rites et pratiques religieuses héritées d'ethnies bien identifiables comme les Yoruba. Les travaux de Roger Bastide sur les religions afro-brésiliennes ont montré que le lien avec le continent africain ne s'était pas rompu en près de trois cents ans de séparation. Les Orixa, les dieux des vieilles religions du golfe du Bénin sont restés sur le continent africain et il faut les appeler pour qu'ils viennent en Amérique, «chevaucher » les corps de leurs fidèles au cours des cérémonies de possession. Il existe même un mythe symbolique d'un arbre dont les racines traverseraient l'océan pour joindre les deux mondes : ce serait le long de ces racines que les Orixa reviendraient quand on les appelle. De même les âmes des morts utiliseraient ces racines pour retourner sur la terre ancestrale alors que leurs corps

\footnotetext{
${ }^{5}$ Christine Chivallon, La diaspora noire des Amériques, réflexion sur le modèle d'hybridité de Paul Gilroy, L'Homme, $n^{\circ} 161, p 60$.
} 
seraient enterrés près des candomblés, «petits morceaux d'Afrique plantés en plein cour du Brésil. Non de l'Afrique profane mais de l'Afrique mystique $»^{6}$.

On ne saurait trouver plus belle illustration à la théorie des diasporas en rhizome.

\section{D'un usage banalisé à la construction d'une catégorie de l'action publique}

Ce que l'usage commun et actuel du terme retient de ce concept dans ces deux acceptations principales est essentiellement le lien qui peut rassembler des gens à partir d'une origine commune plus ou moins lointaine et imprécise mais qui ne suffit pas à elle seule à créer de la dynamique de rassemblement. C'est moins le fait d'avoir un territoire de référence que de vivre loin de celui-ci en partageant d'autres traits identitaires : professionnels, générationnels, religieux, idéologiques qui ferait la diaspora de «sens commun »...La possibilité de créer à partir de là de multiples réseaux sociaux déterritorialisés grâce à la toile permet de décliner à l'infini des références diasporiques qui semblent de prime abord plutôt folkloriques et s'appuient sur plusieurs traits communs. Un coup d'œil sur internet permet d'apprendre l'existence de diaspora de toutes sortes comme la diaspora des rugbymen camerounais en Europe ou la diaspora des cafetiers bretons de New York! Dans un univers globalisé, le recours à la notion de diaspora permet de créer un type de lien bien adapté au contexte. Cet intérêt pour le terme diaspora n'est pas seulement l'affaire des gens ordinaires. Les institutions font aussi de plus en plus référence à la notion de diaspora. Les Etats, les régions, les grandes institutions internationales usent du terme de diaspora pour désigner ceux de leurs ressortissants ou de leurs fidèles qui se sont éloignés et dispersés. Loin d'évoquer aujourd'hui la souffrance d'un exil subi et la nostalgie douloureuse du pays perdu, le mot diaspora est dans le contexte actuel plutôt connoté positivement. Il met en valeur la richesse humaine acquise par le contact avec des univers différents. Il exprime une adaptation heureuse aux réalités d'un monde globalisé.

La mobilisation des diasporas devient un enjeu pour le développement des zones qui restent en retard par rapport au reste du monde. En 2000 a été créée le Haut Conseil de la Diaspora Indienne dont le site milite pour l'obtention de la double nationalité pour les Indiens de l'extérieur. Plusieurs états africains : Maroc, Sénégal, Mali, Niger ont créé des sites dédiés à leurs diasporas afin de les mobiliser pour apporter une contribution au développement du pays. Afin de dépasser les frontières nationales, la banque mondiale a mis en place en 2007 un programme intitulé « Diaspora africaine » qui vise à recenser les personnes d'origine africaine vivant en dehors du continent et qui désirent contribuer à son développement et à la construction de l'Union africaine, quelles que soient leurs nationalités.

En France, si l'on veut bien considérer que le lien diasporique s'exprime avant tout par les initiatives prises en direction du pays d'origine, on peut faire le constat que celles-ci existent depuis longtemps et sont nées de la seule volonté des immigrés qui, dès les années 1970, créèrent des associations pour collecter des fonds et les investir dans des projets de développement dans leurs villages d'origine. Cette migration, composée de ruraux illettrés venus en France pour exercer des métiers peu qualifiés, a réussi à doter les zones d'origine de

\footnotetext{
${ }^{6}$ R.Bastide, Le Candomblé de Bahia, transe et possession du rite du Cadomblé, p 91, Terre Humaine, Poche.
} 
toute une série d'équipements facilitant la vie quotidienne et, en mobilisant des aides des collectivités locales françaises et d'autres institutions, est parvenue à engager des investissement qui se traduisent aujourd'hui par des productions agricoles et des activités de services qui permettent largement de couvrir les besoins des populations locales et de dégager des excédents. De nombreux travaux ont été consacrés à ces initiatives qui témoignent d'un lien diasporique très centré sur les zones d'origine précises. ${ }^{7}$

Alors qu'il y a aujourd'hui une deuxième génération née et éduquée en France issue de cette migration de travail des années 1960 et 1970, on peut se poser la question de la transmission de cette conscience diasporique. A partir d'enquêtes réalisées dans les années 2010 il apparaît que la transmission est très partielle et relativement fragile ${ }^{8}$. D'abord tous les parents ne transmettent pas de lien avec l'Afrique. Certains rejettent même leur pays d'origine qu'ils ont parfois quitté dans des circonstances tragiques. Ceux qui gardent le lien avec le pays d'origine et y réalisent des investissements en vue de son développement sont souvent représentatifs des migrations de travail venues d'Afrique de l'ouest. Même si des efforts sont faits par cette première génération pour associer les jeunes à leurs efforts en direction du pays d'origine, la transmission n'est pas toujours au rendez-vous. Au sein d'une même fratrie il y a des sensibilités très différentes envers cette incitation à s'engager dans l'aide à l'Afrique. Si certains considèrent qu'ils ont un devoir de poursuivre l'œuvre engagée par leurs pères, d'autres ne se sentent pas concernés par un pays d'origine qu'ils connaissent mal ou dans lequel ils ont pu vivre des expériences négatives qui leur ont fait prendre conscience que leur identité était avant tout française.

On observe cependant, chez des jeunes souvent diplômés et qualifiés l'émergence d'un fort intérêt pour l'Afrique mais sans que celui-ci se centre sur la région ou même le pays d'origine de leurs parents. Une initiative comme celle de welcoming diasporas, fondée par des économistes français et africains vise à mobiliser les entrepreneurs, les cadres et les étudiants d'origine africaine en France pour les aider à investir des capitaux en Afrique ou à s'y engager professionnellement. Les sondages réalisés auprès des entreprises dirigées par des personnes d'origine africaine en France révèlent un élargissement considérable des pays qui suscitent l'intérêt. Il ne s'agit plus seulement des pays d'origine des parents mais de tous les pays où il peut y avoir des opportunités, qu'ils soient d'ailleurs anglophones ou francophones.

Est-on pour autant face à une seule volonté de faire des affaires en fonction d'intérêts essentiellement mercantiles et la dimension altruiste et solidaire qui était au cœur du lien diasporique a-t-elle disparue ? Auquel cas on ne pourrait plus pour cette génération parler de diaspora africaine mais plutôt d'une composante de la population française qui exprime un intérêt économique pour le continent africain.

Les choses sont toutefois plus complexes. Les sondages auprès des jeunes entrepreneurs et cadres d'origine africaine traduisent un intérêt pour des domaines qui ont un impact réel sur le développement et relèvent encore d'une éthique solidaire. Ceux-ci veulent agir pour la préservation de l'environnement par des investissements dans le reboisement pas exemple,

\footnotetext{
${ }^{7}$ Voire en particulier Christophe Daum, Quand les immigrés construisent leurs pays, L'Harmattan, 1993.

${ }^{8}$ J.Barou (dir) De l'Afrique à la France, d'une génération à l'autre, Armand Colin, 2011, Paris.
} 
agir en faveur de la lutte contre la pauvreté ou encore contre les violences faites aux femmes. Certes il n'y a rien d'original par rapport aux engagements qui peuvent être ceux de jeunes européens sensibles à toutes ces thématiques. En ce sens on pourrait plus parler d'une « diaspora de cœur » que d'une diaspora d'origine.

Jacques Barou

Directeur de recherche émérite CNRS 